\title{
Field trials of a single dose of the combination rifampicin-ofloxacin-minocycline (ROM) for the treatment of paucibacillary leprosy
}

\author{
M. D. GUPTE \\ National Institute of Epidemiology, Chetput, Chennai, India
}

\section{Trial of a single dose of ROM for single-lesion leprosy}

Single-lesion leprosy poses a unique problem for India in view of the adopted strategy of active case-detection. The proportion of single-lesion patients among the newly detected cases varies among states from 5\% to more than 50\%. It must be noted that, in a sizeable proportion of patients, single-lesion leprosy is thought to undergo spontaneous self-healing. In addition, there are problems regarding diagnostic specificity of this variety of leprosy. It was considered quite acceptable to conduct a trial of single-dose therapy with the combination rifampicin-ofloxacin-minocycline (ROM) among patients with single-lesion paucibacillary (PB) leprosy; rifampicin is strongly bactericidal, and the concurrent administration of ofloxacin and minocycline was expected to prevent selection of rifampicin-resistant mutant Mycobacterium leprae. Therefore, a large multicentre field trial involving 1483 PB leprosy patients with single lesions was conducted in India to study the efficacy of a single dose of ROM. ${ }^{1}$ The trial, sponsored by the World Health Organization (WHO), was a placebocontrolled, randomized, double-blind study involving nine different centres in India.

The study regimen, ROM, consisted of a single dose of $600 \mathrm{mg}$ rifampicin together with $400 \mathrm{mg}$ ofloxacin and $100 \mathrm{mg}$ minocycline; children were administered proportionately smaller doses. The control regimen, the standard WHO regimen for PB leprosy, consisted of a 6-month course of $600 \mathrm{mg}$ rifampicin monthly together with $100 \mathrm{mg}$ dapsone daily. In this case, also, children were administered appropriately smaller dosages. The patients were to be treated for 6 months and followed for an additional 12 months.

Previously untreated patients with only a single skin lesion, whose skin smears were negative, and who were without nerve trunk involvement, were selected for the study. Of the patient above 5 years of age, 745 were male and 729 female; patient intake was completed in July 1995. The measures of outcome selected were 'soft', consisting of clinical improvement, disappearance of lesions and reduction of the clinical score. Ninety-four dropouts were distributed equally between the two treatment groups.

It was possible to follow 1381 patients for 18 months. As shown in Table 1, clinical improvement of the same extent was observed in both treatment groups. Of the 697 patients who had been administered ROM, 51.8\% demonstrated marked improvement, whereas, of the 684 patients who had been administered the standard WHO regimen for PB leprosy, $57.3 \%$ showed marked improvement; this difference was statistically significant. The two 
Table 1. Comparison of ROM and WHO PB MDT in the single-lesion trial

\begin{tabular}{lccc}
\hline & ROM & WHO PB MDT & $P^{*}$ \\
\hline Sample size & 744 & 739 & \\
Followed-up 18 months & 697 & 684 & \\
Clinical improvement (\%) & $99 \cdot 1$ & $99 \cdot 1$ & 1 \\
Marked improvement (\%) & $51 \cdot 8$ & $57 \cdot 3$ & $0 \cdot 04$ \\
Complete cure (\%) & $46 \cdot 9$ & $54 \cdot 7$ & $0 \cdot 004$ \\
\hline
\end{tabular}

\footnotetext{
* The probability that the observed difference between the two treatmentgroups occurred by chance.
}

regimens were also compared with respect to complete cure; $46.9 \%$ of those administered ROM and $54.7 \%$ of those administered the standard WHO PB regimen demonstrated complete cure, a significant difference.

Self-healing of single-lesion leprosy and questionable diagnostic specificity, which may be considered confounding factors, would be expected to lead to an underestimate of the efficacy of the standard regimen in comparison to that of ROM. Even so, the results of treatment by ROM were still very promising. On the basis of these promising results, the National Leprosy Eradication Programme decided to employ a single dose of ROM as standard treatment for Indian patients with single-lesion leprosy.

\section{Trial of a single dose of ROM for PB leprosy with only two or three lesions}

After successful completion of the trial of single-dose ROM for single-lesion patients, the decision was taken to apply almost the same protocol in a WHO-sponsored, multicentre, double-blind trial of single-dose ROM and standard WHO PB MDT for 6 months among PB

Table 2. Comparison of ROM and WHO PB MDT in the trial among patients with two or three lesions

\begin{tabular}{lccc}
\hline & WHO PB MDT & ROM & $P^{*}$ \\
\hline Sample size & 118 & 118 & \\
$\quad$ Two lesions & 82 & 87 & \\
Three lesions & 36 & 31 & \\
18 months follow-up & 103 & 104 & \\
Initial Mean Clinical Score & $26 \cdot 7$ & $26 \cdot 6$ & \\
Clinical improvement (\%) & $96 \cdot 1$ & $96 \cdot 1$ & $1 \cdot 0$ \\
Marked improvement (\%) & $53 \cdot 4$ & $46 \cdot 2$ & $0 \cdot 3$ \\
Complete cure (\%) & $45 \cdot 6$ & $37 \cdot 5$ & $0 \cdot 3$ \\
$\quad$ Two lesions & $42 \cdot 9$ & $39 \cdot 5$ & $0 \cdot 8$ \\
$\quad$ Three lesions & $51 \cdot 5$ & $32 \cdot 1$ & $0 \cdot 2$ \\
One body part involved & $44 \cdot 2$ & $44 \cdot 6$ & $0 \cdot 9$ \\
Two or three body parts involved & $50 \cdot 0$ & $20 \cdot 0$ & $0 \cdot 04$ \\
& & & \\
\hline
\end{tabular}

\footnotetext{
* Probability that the observed difference between the two treatment groups occurred by chance.
} 
patients with only two or three lesions. For this trial, 236 patients, 163 adults and 73 children, were recruited between October 1995 and April 1996. Ninety-two percent of the patients completed 6 months of treatment, and approximately $90 \%$ of the patients were followed for 18 months. The patients who did not complete treatment were equally distributed between the two regimens. As shown in Table 2, clinical scores at intake were very similar among the patients of both treatment groups, and decreased to about the same degree in both groups; the differences observed were not statistically significant at any interval. Approximately 50\% of the patients of both groups showed marked clinical improvement, and complete clearance of lesions was observed in approximately $40 \%$ of the patients of both treatment groups; here, too, the observed differences were not statistically significant. Four patients of each group were judged to represent treatment failure, because they demonstrated no clinical improvement, were found to be smear-positive, or exhibited new lesions. Thus, although the sample size for this trial was much smaller than that for the single-lesion trial, the results were similar.

Additional analyses of various subgroups of patients, also summarized in Table 2, are worth mention. Among patients with involvement of only a single body part, the efficacy of standard WHO PB MDT was very similar to that of ROM, as judged by complete clearance of lesions. However, when two or more body parts were affected, the difference between the regimens was remarkable. Of the 26 patients with involvement of two or more body parts who were treated by standard WHO PB MDT, 13 had complete clearance, compared with only six of 30 such patients who were administered ROM. Thus, if two or more body-parts are affected, i.e. the disease is more extensive, standard WHO PB MDT may be indicated. However, because the number of patients included in this trial is small, no definite conclusions should be drawn.

\section{Single dose for all PB leprosy patients}

In both of these trials, follow-up of patients was continued for only 18 months. Moreover, the sample size, particularly in the trial of patients with two or three lesions, was not very large. For these reasons, we have no information on relapses following treatment by ROM. However, this information is vital.

The possibility was also considered of prescribing the same regimen for all PB leprosy patients. In leprosy control programmes, the majority of all the newly diagnosed PB patients demonstrate from one to three lesions, whereas only a small proportion of the patients demonstrate more than three lesions. Therefore, it was decided to undertake a new trial among all PB leprosy patients with from two to five skin lesions. Of 1592 patients who were recruited for this WHO-sponsored trial between April 1998 and October 1999, about $95 \%$ have completed six months treatment. In addition, approximately 1500 patients with single lesions were recruited for an open trial of ROM therapy. The total duration of the study will be 4 years. We expect these two trials to generate information on both cure and relapse rates.

\section{Acknowledgement}

This study received financial support from the UNDP/World Bank/WHO Special Programme 
for Research and Training in Tropical Diseases (TDR), and was co-ordinated by the Action Programme for the Elimination of Leprosy, WHO, Geneva.

\section{Reference}

1 Single-lesion Multicentre Trial Group. Efficacy of single dose multidrug therapy for the treatment of single-lesion paucibacillary leprosy. Ind J Lepr, 1997; 69: 121-129.

\section{DISCUSSION}

Dr Noordeen: Can you obtain follow-up data, now, 5 years after the first trial that you described?

Dr Gupte: Except for one centre, the patients have not been maintained under surveillance, so that follow-up is not possible.

Dr Sow: In the case of those patients administered the single dose of ROM, was other treatment administered during the trial? Finally, how do you define 'complete cure'?

Dr Gupte: 'Complete cure' means complete disappearance of the lesion or scarformation. The patients administered the single dose of ROM were also administered placebo during the remainder of the trial, so that no patient knew with what he was treated. Unless the outcome was defined as a 'treatment failure', in which case the patient was administered a full, 6-month course of WHO PB MDT, no other treatment was administered. 